\title{
Primary intestinal lymphangiectasia with lymphedema of lower extremities
}

\author{
Kamil Klimas, Katarzyna Drożdż, Angelika Chachaj, Andrzej Szuba \\ Department of Angiology, Hypertension and Diabetology, Wroclaw Medical University, Wroclaw, Poland
}

\begin{abstract}
Primary intestinal lymphangiectasia (PIL), also known as Waldmann's disease, is a rare disorder manifested by the presence of dilated intestinal lymphatic ducts and leading to protein-losing enteropathy. PIL usually presents early in childhood; however, rarely may be also diagnosed in adults. Suggestive laboratory findings include hypoproteinemia, hypoalbuminemia, and hypogammaglobulinemia. Peripheral pitting edema due to hypoalbuminemia is the main clinical feature. Peripheral lymphedema is a less common symptom. We present a case of a 23-year-old woman with lymphedema of lower extremities and PIL diagnosed in childhood.
\end{abstract}

Key words: lymphatics, lymphedema, protein-losing entheropathy, hypoalbuminemia

Acta Angiol 2020; 26, 4: |40-143

\section{Introduction}

Primary intestinal lymphangiectasia (PIL), also known as Waldmann's disease, is a rare disorder, resulting from the presence of dilated intestinal lymphatic ducts and leading to protein-losing enteropathy. Dilated intestinal lymphatics lead to lymph leakage into the lumen of the small intestine and consequently, to hypoalbuminemia, hypogammaglobulinemia, lymphopenia, and in severe cases to generalized edema. PIL usually occurs in children before 3 years of age and adolescents, but rarely may be also diagnosed in adults [ $[1,2]$. We described a case of a 26-year-old woman with lymphedema of lower extremities and PIL diagnosed in childhood.

\section{Case report}

A 26-year-old woman was referred to the Department of Angiology, Hypertension and Diabetology at Wroclaw Medical University because of edema of lower extremities. The lower limb edema was observed for the first time at the age of six months and has gradually progressed. The medical history of the patient revealed a very typical course of primary intestinal lymphangiec- tasia (PIL). Shortly after birth, temporary swelling of the labia appeared. The edema of the lower extremities was the next symptom. Until the age of five, the patient was repeatedly hospitalized due to recurrent respiratory infections accompanied by hypoalbuminemia $(2 \mathrm{lg} / \mathrm{L})$, hypoproteinemia $(42 \mathrm{~g} / \mathrm{L})$, hypogammaglobulinemia (IgG: 2-3.5 g/l; lgA: $0.16 \mathrm{~g} / \mathrm{l}$ ) and lymphopenia (10\%). The endoscopic examination of the gastrointestinal tract and albumin scintigraphy did not clearly confirm the PIL. The abdominal computed tomography (CT) and magnetic resonance imaging (MRI) have shown small intestine thickening with slight ascites. Finally, at the age of five, the patient underwent a laparotomy, which revealed the presence of intestinal lymphangiectasia. Histopathological examination of biopsy specimens taken during laparotomy established the suspected diagnosis of PIL. Since childhood the patient did not suffer from any recurrent abdominal pain, nausea, vomiting, weight loss, obstructive ileus, or steatorrhea. The lower extremity edema during the current hospitalization was the dominant clinical symptom, which significantly affected the everyday life of the patient (Fig. I). Diet therapy with medium-chain triglycerides (MCT) has been applied since the diagnosis of PIL. In the past, the 


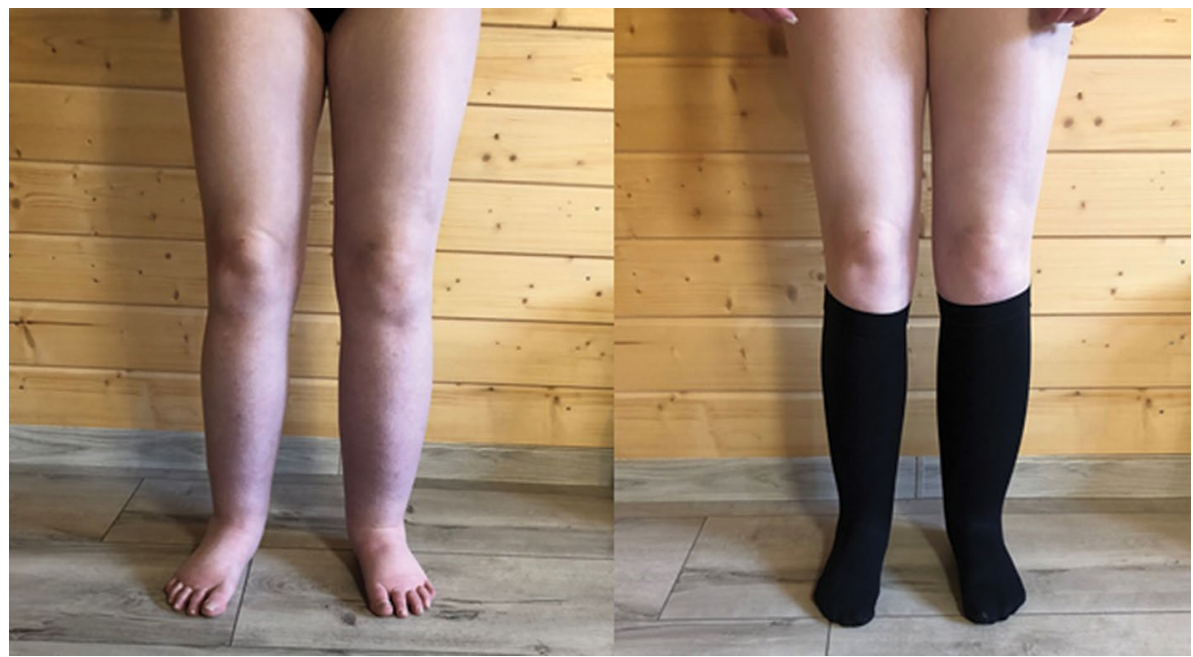

Figure I. Lymphedema of the lower extremities in our patient. In the second photo the patient is wearing the compression stockings, which are the basic treatment of lymphedema

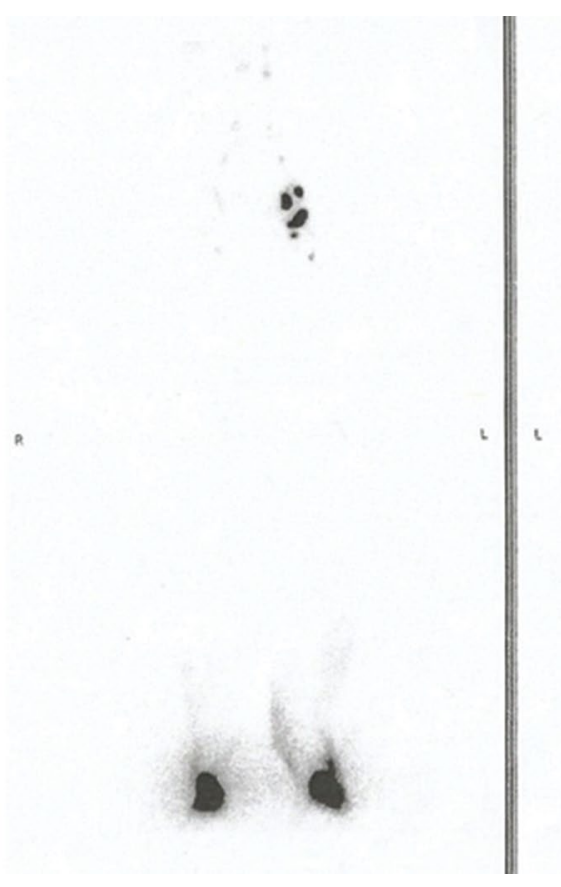

Figure 2. Lymphoscinthigraphy showing the lack of visualization of the lymphatic trunks in both legs and of the inguinal lymph nodes on the right side. Dermal backflow is also visible within the calves

patient was also treated with intravenous immunoglobulins, intravenous fresh frozen plasma, and octreotide. However, these therapies resulted in only temporary improvement of hematological and scintigraphy findings. The patient has never been treated with compression therapy because of the leg lymphedema.

On a physical examination performed during the current hospitalization, the scar after laparotomy and moderate peripheral edema of lower extremities, with Stemmer's sign and skin thickening, was noted. The laboratory studies revealed hypoalbuminemia and hypogammaglobulinemia. The lymphoscintigraphy of the lower extremities revealed the lack of visible lymphatic trunks in both legs and of the inguinal lymph nodes on the right side. Dermal backflow was also noticeable within the calves (Fig. 2). The compression therapy, 
firstly with low-stretch bandages and secondly with compression stockings, has been started with the good clinical effect of lymphedema reduction. The sirolimus, a mammalian target of rapamycin (mTOR) inhibitor and/or intestine microsurgery has been considered for further treatment.

\section{Discussion}

Primary intestinal lymphangiectasia (PIL) is a rare protein-losing enteropathy of unknown prevalence. Less than 200 cases have been reported since 196I when Waldmann et al. described the first 18 cases of the disease [I]. The etiology of PIL is unknown. It is caused by congenital malformation or obstruction of intestine lymphatics with concomitant increased intraluminal pressure. The disease can affect all small intestine or maybe segmental. It leads to dilatation of lymphatic vessels in mucosa, submucosa, and subserosa and lymph leakage into the small bowel lumen resulting in hypoalbuminemia, hypogammaglobulinemia, and lymphopenia $[1,2]$. The basic clinical feature is peripheral pitting edema of variable degree, usually symmetrical, due to low oncotic pressure usually involving lower limbs, face, external genitalia, scrotum, or vagina. Serous effusions in more severe cases are also common - pleural effusion, chylous ascites, and pericarditis may be present in patients and may be life-threatening. Other concomitant symptoms are abdominal pain, nausea, vomiting, moderate diarrhea, fat-soluble vitamin deficiencies, and weight loss [2-5]. In our patient, there were no digestive symptoms all the time. However, diet therapy was applied just after the diagnosis of PIL. The swelling of the labia was the earliest symptom of the disease in our patient. Lymphedema of lower extremities was the next clinical feature. Lymphedema of lower limbs is a rarer symptom in PIL [I]. Lymphedema of upper extremities, breast, and external genitalia were also noted [6]. At the age of 26, lymphedema of lower limbs affected our patient's quality of life the most. However, compression therapy was started scarcely during hospitalization in our Department.

The diagnosis of PIL in our patient was based on the clinical course, laparotomy, and histopathological findings of intestine specimens taken during laparotomy. At present, endoscopy with histopathological assessment of intestine biopsy is the most important tool in PIL diagnostics [I]. The laboratory findings of hypoproteinemia, hypoalbuminemia, hypogammaglobulinemia, and lymphocytopenia may be suggestive. The macroscopic abnormalities which may be found during small bowel endoscopy include scattered whitish spots (snowflake appearance), thickened villi, and edematous mucosa of the small intestine [7]. Although capsule endoscopy cannot be used to perform a biopsy, it is a comfortable method to examine mucosa of the small intestine $[1,7,8]$. Radiologic examinations also can be useful. Ultrasonography has the potential to show dilation of the intestinal loops, regular and diffuse thickening of the walls, plical hypertrophy and mesenteric edema [9]. CT may reveal thickening and edema of small intestine wall, as a consequence of the dilated lymphatics [10, II]. Moreover, Sun et al. documented a new diagnostic method of multiple-detector computed tomography (MDCT) after direct lymphangiography in 55 patients with PIL. In this study, intra- and extraintestinal abnormalities, especially regarding the morphology and the extent of lymphatic vessel involvement were observed. Therefore, it can be a significant tool for the diagnosis of PIL [1 2]. Lymphoscintigraphy is a useful diagnostic tool for identifying abnormal lymphatics within the upper and lower extremities [I, I3].

The rarity of PIL is the reason for the lack of treatment guidelines in this disorder. Dietary intervention is the gold standard in PIL management. A low-fat and high-protein diet supplemented with medium-chain triglycerides (MCT) is usually recommended $[7,8]$. The majority of PIL children respond to dietary therapy and according to Prasal et al. only $20 \%$ need additional therapy [14]. Lipid elements in food provoke lymphatic pressure increase and lymphatic leakage into the small bowel lumen. A low-fat diet reduces lymphatic flow and lacteal dilation and thus helps to prevent lymph loss. MCTs are absorbed directly in portal venous circulation by passing the lymphatic system and thus provide nutrient fat and lessens lacteal engorgement [2]. Other treatment methods can be used in the combination with a low-fat diet with MCT supplementation, mostly in severe cases. These are as follows: octreotide (I 50-200 ug twice a day or slow-release formulation), tranexamic acid ( I g, 3 times a day), antiplasmin, corticosteroids, vitamin $D$ supplementation, albumin infusion (applied in patients with significant serous effusion) $[1,15]$. In the cases with segmental lesions within intestine, surgical procedures as small bowel resection may be recommended [16]. Recently, the successful use of mTOR inhibitors (sirolimus, everolimus) in the treatment of a small collection of vascular anomalies and PIL has also been reported [17, 18]. Lymphedema of upper and lower extremities should be treated with standard compression therapy, including complex physical therapy and compression garments [19].

\section{Conflict of interest}

None. 


\section{References}

I. Vignes S, Bellanger J. Primary intestinal lymphangiectasia (Waldmann's disease). Orphanet J Rare Dis. 2008; 3: 5, doi: 10.1 186/1750-1 I72-3-5, indexed in Pubmed: 18294365.

2. Ingle $S B$, Hinge Ingle CR. Primary intestinal lymphangiectasia: Minireview. World J Clin Cases. 2014; 2(10): 528-533, doi: 10.12998/wjcc.v2.il 0.528, indexed in Pubmed: 25325063.

3. Lopez RN, Day AS. Primary intestinal lymphangiectasia in children: A review. J Paediatr Child Health. 2020; 56(II): 1719 1723, doi: 10.1 I I I/jpc. 14837, indexed in Pubmed: 32463559.

4. Abramowsky C, Hupertz V, Kilbridge P, et al. Intestinal lymphangiectasia in children: a study of upper gastrointestinal endoscopic biopsies. Pediatr Pathol. 1989; 9(3): 289-297, doi: 10.3109/15513818909037733, indexed in Pubmed: 2748490.

5. Lee WS, Boey CC. Chronic diarrhoea in infants and young children: causes, clinical features and outcome. J Paediatr Child Health. 1999; 35(3): 260-263, doi: 10.1046/j.14401754.1999.00356.x, indexed in Pubmed: 10404446.

6. Goktan C, Pekindil G, Orguc S, et al. Bilateral breast edema in intestinal lymphangiectasia. Breast J. 2005; I I(5): 360, doi: I0. I I I //. I075I22X.2005.21578.x, indexed in Pubmed: 16174162.

7. Wen J, Tang $\mathrm{Q}, \mathrm{Wu}$ J, et al. Primary intestinal lymphangiectasia: four case reports and a review of the literature. Dig Dis Sci. 2010; 55(I2): 3466-3472, doi: I0.1007/s 10620-010-II6I-I, indexed in Pubmed: 20198428.

8. Lee SJ, Song HJ, Boo SJ, et al. Primary intestinal lymphangiectasia with generalized warts. World J Gastroenterol. 2015; 21 (27): 8467-8472, doi: 10.3748/wjg.v21.i27.8467, indexed in Pubmed: 26217101.

9. Maconi G, Molteni P, Manzionna G, et al. Ultrasonographic features of long-standing primary intestinal lymphangiectasia. Eur J Ultrasound. 1998; 7(3): 195-198, doi: 10.1016/s09298266(98)00037-8, indexed in Pubmed: 9700215.

10. Mazzie J, Maslin P, Moy L, et al. Congenital intestinal lymphangiectasia. Clinical Imaging. 2003; 27(5): 330-332, doi: I0.1016/ s0899-707I(02)00588-0.
II. Yang DMo, Jung DH. Localized intestinal lymphangiectasia: CT findings. AJR Am J Roentgenol. 2003; 180(I): 2/3-2/4, doi: 10.22 I4/ajr.180.1.1800213, indexed in Pubmed: 12490507.

12. Sun $X$, Shen $W$, Chen $X$, et al. Primary intestinal lymphangiectasia: Multiple detector computed tomography findings after direct lymphangiography. J Med Imaging Radiat Oncol. 2017; 6I (5): 607-6 13, doi: 10.1 II I/1754-9485. 12606, indexed in Pubmed: 28345300 .

13. Szuba A, Shin WS, Strauss HW, et al. The third circulation: radionuclide lymphoscintigraphy in the evaluation of lymphedema. J Nucl Med. 2003; 44(I): 43-57, indexed in Pubmed: I25I5876.

14. Prasad D, Srivastava A, Tambe A, et al. Clinical profile, response to therapy, and outcome of children with primary intestinal lymphangiectasia. Dig Dis. 2019; 37(6): 458-466, doi: 10.1 159/000499450, indexed in Pubmed: 31030202.

15. Sari S, Baris Z, Dalgic B. Primary intestinal lymphangiectasia in children: is octreotide an effective and safe option in the treatment? J Pediatr Gastroenterol Nutr. 20 I0; 5 I (4): 454-457, doi: 10.1097/MPG.0b0/3e3l8Idlbl62, indexed in Pubmed: 20512058.

16. Chen CP, Chao Y, Li CP, et al. Surgical resection of duodenal lymphangiectasia: a case report. World J Gastroenterol. 2003; 9(I2): 2880-2882, doi: $10.3748 / \mathrm{wjg} . v 9 . \mathrm{i} \mid 2.2880$, indexed in Pubmed: 14669360.

17. Hammill AM, Wentzel M, Gupta A, et al. Sirolimus for the treatment of complicated vascular anomalies in children. Pediatr Blood Cancer. 201 I; 57(6): 1018-1024, doi: 10.1002/ pbc.23।24, indexed in Pubmed: 21445948.

18. Ozeki M, Hori T, Kanda K, et al. Everolimus for primary intestinal lymphangiectasia with protein-losing enteropathy. Pediatrics. 2016; 137(3): e20152562, doi: 10.1542/peds.2015-2562, indexed in Pubmed: 26908672.

19. Szuba A, Rockson SG. Lymphedema: classification, diagnosis and therapy. Vasc Med. 1998; 3(2): 145-156, doi: 10.1 I77/1358836X9800300209, indexed in Pubmed: 9796078. 